\title{
High Resolution Monochromated EELS Really Is Different!
}

\author{
Peter Rez ${ }^{1}$, T. Aoki ${ }^{2}$ \\ 1. Peter Rez, Department of Physics, Arizona State University, Tempe, AZ 85287-1504, USA \\ 2. Toshihiro Aoki, LE-CSSS, Arizona State University, Tempe, AZ 85287-1704, USA
}

Until recently analytical electron microscopy was limited to the X-ray and UV parts of the spectrum. With $\mathrm{LaB}_{6}$ guns, the energy resolution was about $1 \mathrm{eV}$, which improved to about $0.5-0.8 \mathrm{eV}$ with the introduction of thermally assisted field emission guns. Although in principle it should have been possible to detect excitations in the optical region, $1.8-2.3 \mathrm{eV}$, in practice the extended tail of the zero loss made it very difficult, especially for excitations associated with defects. Use of cold field emission guns improved energy resolution to about $0.4 \mathrm{eV}$ and monochromators in the electron gun narrowed the zero loss peak to about $0.1 \mathrm{eV}$. These capabilities made it possible to explore optical excitations, and found prominent application in studies of plasmonic effects, but the IR region still remained out of reach. The combination of monochromator and spectrometer on the Nion UltraSTEM has narrowed the zero loss peak to better than $10 \mathrm{meV}$, and has made it possible to do IR spectroscopy at the nanoscale.

What makes these new capabilities so different? Given that lifetime broadening on inner shells is about $80 \mathrm{meV}$, even for low energy excitations like $\mathrm{Si}_{23}$, there is nothing to be gained from using high resolution monochromated EELS. The interesting feature are the vibrational modes in the IR and optical excitations from defects. An energy resolution of $10 \mathrm{meV}$ or less makes it possible to detect all the peaks from stretching of hydrogen bonded to other elements, and also characteristic peaks from bond stretches in carbonates, silicate, phosphates and nitrates. Since there is only energy loss, not energy gain (except for low energy acoustic phonons), there is no significant multiple scattering background in the IR region or the optical region of insulators. In contrast to inner shell spectroscopy, thickness is not the problem. Larger thicknesses are good and longer path lengths in aloof beam mode (beam outside the specimen) make it possible to detect features that have low scattering cross sections, or features from isolated defects.

When recording inner shells one could assume that the signal came from a single atomic column or the volume illuminated by the electron probe. "Delocalization" over a range of a few nm was a concern for UV excitations such as plasmons or interband transitions in insulators but since the states were delocalized anyway it wasn't a major limitation. The delocalization increases as the energy loss is reduced. In aloof beam spectroscopy of vibrational modes or optic phonons it is possible to record signals when the electron probe is $100 \mathrm{~nm}$ or more away from the edge of the specimen. This clearly lowers the spatial resolution but there is also a benefit. The probe can be positioned far enough away from the specimen to avoid exciting higher energy excitations that break bonds and lead to radiation damage, while still recording vibrational mode spectra. This capability has been used to good effect in measuring the peaks due to hydrogen in both inorganic and biological specimens. Figure 1 shows how it is possible to record all the peaks related to vibrations of hydrogen attached to other atoms in a beam sensitive biological specimen in aloof mode with an electron probe as far as 100nm from the specimen edge.

The delocalized signal arises from an oscillating dipole due to different effective charges on atoms making up the bonds in solid. There is also a localized signal, the impact scattering, similar to that from 
acoustic phonons. In principle it should therefore be possible to record signals from vibrations in a solid at atomic resolution.

The narrowing of the zero loss peak is important not just for resolving features close in energy, but also for lowering the background from the tail of the zero loss peak. Reducing the zero loss tail to $10^{-5}$ of peak intensity at the energy of an optical excitation makes it possible to detect point defects such as color centers or the NV center in diamond and localize them with nm resolution. It is not just the tail of the zero loss that gives background in band gaps. Cerenkov radiation is a serious problem, especially for semiconductors. The best way to eliminate it is to use an accelerating voltage lower than the Cerenkov threshold. A quick estimate can be found by comparing the electron velocity to the speed of light divided by the optical refractive index (or $\sqrt{\varepsilon_{r}}$ ). It might be possible to subtract Cerenkov losses if the spectrometer disperses in both energy loss and angle. Cerenkov is not just a problem when the beam is on the specimen, it is also present in aloof beam spectroscopy. An image charge in the specimen travels parallel to the electron outside the specimen, and there will be Cerenkov losses if it is travelling faster than the speed of light in the material. Since multiple scattering is not an issue the need to avoid Cerenkov means that in many cases it is better to use lower rather than higher accelerating voltages!

\section{References:}

[1] O.L. Krivanek et al, Nature, 514 (2014) p. 209.

[2] P. Rez et al, Nature Commun., (2016)

[3] T. Aoki, L.A.J. Garvie and P. Rez, Ultramicr.153 (2015), p. 40.

[4] L. Gu et al, Phys. Rev. B 75 (2007) 195214.

[5] We acknowledge use of facilities in the LE-CSSS at ASU and funding from NSF grant CHE 1508667. We also thank Drs P.A. Crozier, C. Dwyer, H. Cohen, L.A.J. Garvie, and O.L. Krivanek for discussions, and Drs N. Dellby, D. Gur, O.L. Krivanek and T.C. Lovejoy for the data of Fig. 1a and Drs K. March and H. Cohen for the data of Figs $1 \mathrm{~b}$ and $1 \mathrm{c}$.

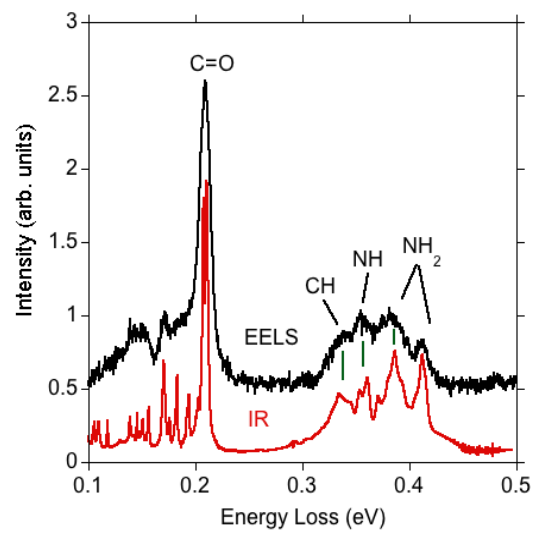

(a)

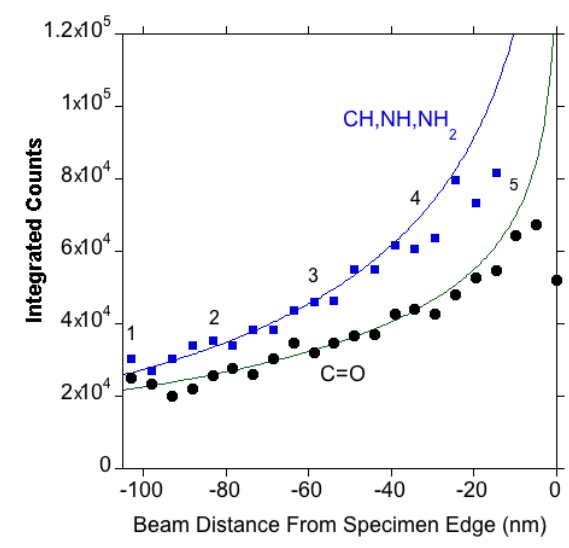

(b)

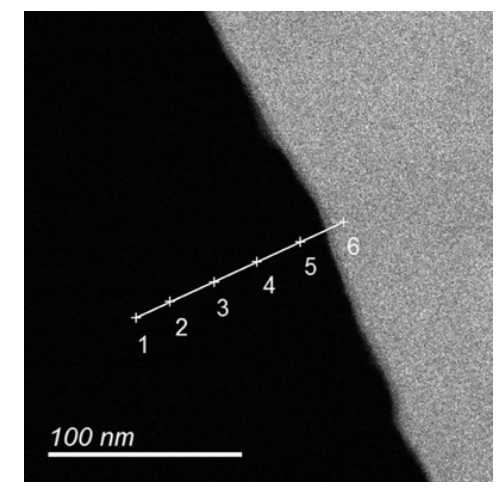

(c)

Figure 1. (a) IR region spectrum from guanine fish scale, (b) Variation of peak intensity with position (c) Image showing line trace for (b) 